\title{
Analysis and Research on Factor Differences in Abnormal Conditions of Indicators
}

\author{
Liu Yun, Shuping Luo, Xiaoyun Zhang* \\ Guangzhou Huashang College, Guangzhou, China \\ Email address: \\ ly2005112@163.com (Liu Yun),78107645@.qq.com (Shuping Luo),773590744@qq.com (Xiaoyun Zhang) \\ *Corresponding author
}

\section{To cite this article:}

Liu Yun, Shuping Luo, Xiaoyun Zhang. Analysis and Research on Factor Differences in Abnormal Conditions of Indicators. Journal of Finance and Accounting. Vol. 9, No. 3, 2021, pp. 67-79. doi: 10.11648/j.jfa.20210903.12

Received: April 28, 2021; Accepted: May 19, 2021; Published: May 24, 2021

\begin{abstract}
In factor variance analysis, the index values of the reporting period and the base period usually appear in the following five situations: the reporting period and the base period are both positive; the reporting period is positive, and the base period is negative; the reporting period is negative, and the base period is positive; Both the reporting period and the base period are negative, and the negative numerator is less than the negative denominator; the negative numerator is greater than the negative denominator. In the first case, the problem has been solved; the latter four cases have not been paid attention to by scholars. In the last four situations encountered in factor variance analysis, we call it indicator anomalies. Based on the factor analysis "index logarithmic ratio" method, we try to use the variation range (variation difference/base period number) logarithmic ratio method to solve. In order to show the reality of the research, we use the ZXGS financial data indicators as the blueprint, use the case method to study the financial analysis of abnormal indicators, and carry out the event analysis procedure anatomy based on the new method of factor analysis and variance analysis. Through the study of these several situations, we hope that the exponential logarithmic ratio method of factor analysis and difference analysis will be more pertinent, complete, adaptable, and scientific, and can be correctly applied in a wide range of social practices.
\end{abstract}

Keywords: Factor Analysis, Variance Analysis, Index Abnormality, Variation Range, Absolute Value, Exponential Logarithmic Ratio Method

\section{Introduction}

Factor analysis and factor variance analysis have a wide range of applications. In the mathematical equation of multi-index combination, all the basic formulas of analysis have a product structure and a quotient structure, which has the problem of incorrect distribution of factor interaction. Mathematics introduces a negative exponent instead of the denominator, so there is no quotient structure, and the quotient structure is also a product structure; this is just like the mathematical equation and difference structure, which introduces the concept of negative numbers, and there is no "difference" (subtraction), a number subtraction To go to another number, we call it "plus a negative number", and the sum and difference structure becomes the sum structure; the same reasoning: the quotient structure becomes the product structure after the negative exponent is introduced. In the overall variance analysis of the product structure, there is a problem of sharing the interaction between factors. The basic mode of factor analysis is mostly mixed structure: sum product structure. In the product structure, when we calculate the change of the overall (primary factor) difference, there is an interaction between the factors in the function formula. For example: the main factor $y=a+b x, \Delta y$, $\mathrm{bx}$ are the product structure, there is an interaction between $\mathrm{b}$ and $\mathrm{x} \Delta \mathrm{b} \cdot \Delta \mathrm{x}$ (the multiplication sign is omitted below); $\mathrm{m}=\mathrm{ab}$, in the $\Delta \mathrm{m}$ analysis, because $\mathrm{ab}$ is the product structure, The change of the factor between $a$ and $b$ has an interaction effect of $\Delta \mathrm{a}^{*} \Delta \mathrm{b}$ on the change of the main factor $\mathrm{m}$. The traditional factor analysis and factor difference analysis methods are: serial substitution method, difference substitution method, and index method (different names in different periods), without considering the interaction between factors. "Journal of Finance and Accounting" (2019.12, see References [30-32]) published an article, from the mathematical mechanism of plane geometry and 
algebraic calculus, demonstrated the existing problems of the factor analysis method and the reasons for the errors; The new solution method of factor analysis "exponential logarithmic ratio" method is deduced. The new solution was put forward and solved the unresolved problem of factor analysis for a long time, and it was endorsed by peer experts and scholars. In the construction of economic and accounting models, if the factors in the basic model are multiplicative, adding explanatory variables for interaction terms in the construction model will improve the goodness of fit of the model (another example).

It must be added that the new method only solves the conventional cases that appear in the textbooks, namely actual and budget, this year and the previous year, the reporting period and the base period, the company and the industry conventional cases; the main feature of the conventional cases is: these data are all Regular positive numbers, that is, the numerator and denominator of the ratio of the exponent are both positive, and the logarithm can only be taken when these ratios are positive. When the numerator and denominator of this kind of change have different signs, or both are negative factor analysis, is the "exponential logarithmic ratio method" of factor analysis and variance analysis unavailable? What this article aims to solve is when the indicators vary in four situations: the numerator is positive and the denominator is negative; the numerator is negative, and the denominator is positive; the numerator and denominator are both negative, and the numerator is greater than the denominator or the numerator is less than the denominator; factors at this time The exponential logarithmic ratio method of analysis and variance analysis, or the logarithmic ratio method of the absolute value of the change ratio.

"Factor Difference Analysis in Abnormal Conditions of Indicators" aims to solve the problem of factor analysis and the application of difference analysis techniques. The business behaviors in the cases cited are not politically inclined or evaluated; starting from the analysis technology level, The case data has the complex conditions for us to implement factor analysis and variance analysis.

This paper is a supplement to the combined paper published in Finance and Accounting by the author in 2019 [Refs. 31, 32, 33]. Research significance: 1. To make the factor analysis, difference analysis of the new method of exponential and logarithmic proportional method application, more comprehensive, complete, systematic, scientific, accurate. 2. It is the conclusion of the fruitless discussion on "Accounting Research" many years ago; It is expected that correct and scientific methods can be widely used in both theory and practice.

\section{Literature Review}

\subsection{Chinese and Foreign Accounting Circles, the Above-mentioned Problems Co-exist}

Based on the relevance of the article, we take the generation and development of propositions, and attribute clustering as the context, and we sort them out as follows:

In current American accounting textbooks, in factor analysis and cost variance analysis, there is a problem of unfair distribution of factor interaction in the multiplication model. Roman L. Weil (Roman L. Weil), Katherine Schipper (Katherine Schipper), Jennifer Francis (Jennifer Francis), Zhu Dan, Qu Tenglong translated. (2015) [2] Financial Accounting-Concepts, Methods and Applications ("Financial Accounting" directly uses the difference of the main factors to analyze the difference, without decomposing the specific influence of each factor on the main factor. Srikant M. Datar (Srikant M. Datar) Madhav $\cdot V$ Rajan (Madhav V. Rajan) "Management Accounting" [M]. (2015) [3] Charles T. Horngren Srikant M. Datar Madhav V. Rajan (2016) [5] in his "Cost and Management Accounting" writing Analyzed the favorable and unfavorable effects of multi-factors on output, product unit consumption, material unit price; labor hours, labor hours unit price, and product time consumption on cost changes. When analyzing the difference in cost, the factor analysis in the product model is used It is the difference substitution method (the essence of the serial substitution method is still the difference substitution method), which does not consider the interaction of factor factors. In the absolute quota index variables, there are additional factor analysis such as "efficiency" and other composite indicators.

Other countries also have problems with the application of factor analysis. Yousef Soar (2018)[34] uses factor analysis to determine the impact of related parties on the quality of financial reporting-taking the Algerian system as an example. Izolda Chiladze (2017) [36] Factor analysis of corporate operating leverage. Kang Ho [37] published a paper: Institutionalization of Management Accounting Reform: An Analysis of Institutionalized Factors Based on my country's Enterprise Activity-Based Costing Method. Muhammad Onsi (1973) [38] took "Analysis of Influencing Factors of Budget Easing Behavior Variables" as the subject, using factor analysis techniques. The factor analysis method is widely used in different countries and different industries [35]; but it is mostly the content factor analysis, not the factor difference analysis; it downplays the problems of the factor analysis method itself.

Related textbooks in my country's accounting circles also have the problem of factor interaction or unfair distribution. Zhang Xianzhi and Chen Youbang "Financial Analysis" (9th edition) [M]. The "Twelfth Five-Year" general higher education undergraduate national-level planning textbook, national-level quality courses (2019) adopts factor analysis and difference substitution method throughout the article [4]. Organized and compiled by the Chinese Institute of Certified Public Accountants, the CPA exam book year CPA national unified exam guidance textbook "Financial Cost Management" (2020) [6] When analyzing the equation quotient structure, the denominator is directly regarded as 1 unchanged, and then the numerator is analyzed. The influence of the main factor is not analyzed. In factor analysis and cost variance analysis, the serial substitution and 
balance substitution methods are used to let individual factors bear the interaction of factors in the product model. Meng Yan, Liu Junyong (2017) [7] "Cost Management Accounting" General Higher Education "Twelfth Five-Year" Undergraduate National Planning Textbook; Wu Xiaoling, Tian Gaoliang Editor-in-Chief (2018) [8] "Corporate Financial Analysis" 21st Century Economics and Management Planning textbook, Peking University Press; Wen Subin "Management Accounting: Theory·Model·Case" (2014) [9] Excellent planning textbook, Machinery Industry Press; Editor-in-chief, Sun Maozhu, Zhi Xiaoqiang, Dai Lu, National Outstanding Achievement Award, The "Twelfth Five-Year Plan" general higher education undergraduate national-level planning textbook "Management Accounting" (2019)[10] cost variance analysis uses the variance substitution method (or serial substitution method). Peng Jiaqing, Zhang Daoyun (2008) [11, 12] Application cases of factor analysis in coal mine production cost and product production cost analysis. Zhao Shundi, Kong Yusheng, Lu Min (2007) [37] Mine cost analysis and cost control based on sensitivity factor analysis. Zhao Banhong, Zhang Zhenchuan, Guo Lihua (2005) [38] Analysis of factors affecting the change of asset appraisal rate of appreciation, etc.; they are all cases of direct application of current factor analysis methods, without involving the issue of factor analysis methods themselves.

\subsection{Tracing the Root of the Problem, It Has a Long History}

The origin of the discussion: After Li Qiyu and Yu Rong's "A New Probe into Factor Analysis" were published in the second issue of "Accounting Research" in 1984, the editorial department of "Accounting Research" received some manuscripts for discussion with the author of the article. In the honor of Zhou Zonglin, the manuscripts were sorted out and published in the journals "Accounting Research" in the 5th issue in 1984 and the 6th issue in 1985.

Ji Liang (1984) [24] "Discussion on the Method of Factor Analysis in "Das Kapital"" The method of factor analysis is a commonly used method in the analysis of economic activities. The basic principle is: when the value is composed of several factors such as a, b and c, that is, abc = $d$, compare the value of $d$ during the reporting period $\left(d_{1}=\right.$ $a_{1} b_{1} c_{1}$ ) with the value of $d$ in the reference period when the difference $\left(\mathrm{d}_{1}-\mathrm{d}_{0}\right)\left(\mathrm{D}_{0}=\mathrm{a}_{0} \mathrm{~b}_{0} \mathrm{c}_{0}\right)$, how much influence does the changes of the three factors $a, b$ and $c$ have. This article traces the origin of factor analysis to Marx's proposition in "Das Kapital", and analyzes the influence of a single factor change and other factors unchanged on surplus value. Ji Liang traced the case of factor analysis back to Marx's "Das Kapital". When Marx used factors to analyze the impact of capital composition structural factors on total capital, he often analyzed the impact of one or two factors on capital and surplus value, and ordered other factors. The factors remain unchanged; then analyze them one by one. It does not enumerate the cases where all the factors have changed on the main factors.

Zhou Zonglin (1985) [23] "Excerpts from Discussions
on'Decomposition Methods' No. 2" After the publication of "Excerpts from Discussions on Decomposition Methods" in the fifth issue of 1984, the editorial department of Accounting Research received a lot of praise In the article, the participants in the discussion expressed various opinions and proposed different methods. Among them, Comrade Zhou Zonglin concluded that the analysis results of the "factor decomposition method" were inaccurate and reasonable, and proposed another factor analysis method. The editorial department of "Accounting Research" has made factor analysis a special topic, but no scholars or experts have solved the substantive problems of factor analysis, and no new methods have been proposed to solve related problems. Li Qiyu and Yu Rong (1984) [25] The "New Exploration of Factor Analysis" series of substitution methods is a basic method used in economic analysis. Since this method has a different order for replacing various factors, it has different impact values. The analysis result has no definite answer. Li Qiyuwen tries to use graphs to solve factor analysis problems. This is a preliminary discussion on factor analysis initiated by the "Accounting Research" editorial department. Chen Yamin (1987) [19] "Discussion on the Basic Principles of Factor Analysis" The factor analysis method is a quantitative analysis method used to study the influence of changes in various factors on the overall index. It is not only widely used in post analysis (such as accounting statement analysis), but also widely used in pre-decision analysis (such as sensitivity analysis) and in-process control analysis (such as difference analysis). Because the substitution method is used more in actual work, it has almost become synonymous with factor analysis in many occasions. However, the two are not the same. Chain substitution method (the original title of "serial substitution method") is just one of the factor analysis methods. As early as the late 1950s, the Chinese accounting community realized that the calculation results of the chain alternative method may be incorrect, and sometimes even incorrect. The article puts forward the existing problems of factor analysis.

Yang Zengwu (1984) [26] "Scientifically question the method of factor analysis in indicators" The method of factor analysis in statistical research indicators often finds theoretical basis from Marx's "Das Kapital". When analyzing the length of the labor day, the impact of labor productivity and labor intensity on the relative quantity of labor price and surplus value, Marx said: "It is obvious that there may be many combinations. Perhaps two factors have changed, one is the factor. Not change, or these three factors change at the same time, they can change to the same or different degrees, and can change in the same direction or in the opposite direction, so their changes can partially or completely offset the others." Yang Wen systematically proposed The factor analysis index method of multiple varieties is introduced. This is an innovative method in addition to the factor analysis method series substitution method and the difference substitution method. However, in the combination of factor products, the factor combination is obviously missing; it cannot solve the 
problem of the ratio of the negative numerator to the negative denominator; it cannot solve the problem of the distribution of interaction. Li Zexuan (1985) [27] "Questions about the scientific problems of the factor analysis method in the index-and on the theory of the three index systems", questioned Professor Yang Zengwu's article, and proposed relative index, relative index and absolute index. There are still problems with factor analysis. At the same time, the theory of the "three-index system" was demonstrated through the three-factor geometric method, but the problem of sharing the interaction between the factors has not been resolved. [28, 29, 30] There are also many articles involved in discussions and factor analysis applications.

In the sixth issue of "Accounting Research" in 1985, Zhou Zonglin published a summary of the topic discussion on the factor decomposition method, which was based on Yang Xiongsheng's point of view: the use of the serial substitution method (the difference substitution method) ended; the subsequent "factor decomposition method" "Discussion essays can be found in the journal "Finance and Accounting". It can be seen that the "discussion of factor decomposition method" that has lasted for many years is unresolved and ended in fruitless. It also cannot solve the problem of interaction distribution. Li Zexuan (1985) [27] "Questions about the scientific problems of the factor analysis method in the index-and on the theory of the three index systems", questioned Professor Yang Zengwu's article, and proposed relative index, relative index and absolute index. There are still problems with factor analysis. At the same time, the theory of the "three-index system" was demonstrated through the three-factor geometric method, but the problem of sharing the interaction between the factors has not been resolved.

In the sixth issue of "Accounting Research" in 1985, Zhou Zonglin published a summary of the topic discussion on the factor decomposition method, which was based on Yang Xiongsheng's point of view: the use of the serial substitution method (the difference substitution method) ended; the subsequent "factor decomposition method" "Discussion essays can be found in the journal "Finance and Accounting". It can be seen that the "discussion of factor decomposition method" that has lasted for many years is unresolved and ended in fruitless.

\subsection{Once a Special Discussion, a Hundred Schools of Thought Contended}

The problems of factor analysis methods were initiated by the "Accounting Research" and "Finance and Accounting" editorial departments in 1984-2000, and initiated fruitful discussions in the field of accounting theory and practice in my country; the editorial department compiled and distributed a large number of factor analysis feature articles, The experts analyzed the causes of the problems and tried various methods to solve the problems.

Li Shanzhai (1997) [13] published a paper, taking the factor analysis of various types of causal relations as an example, discussing and summarizing the regularity and techniques of directly writing out the calculation formula of the influence value of each factor on the analysis object, and trying to calculate the number as a percentage A unified derivation mode of calculation formula. Attempt to replace the current serial substitution method and difference substitution method with the calculation percentage difference analysis method. Zhang Xiuqi (1991) [14] published an article in the journal "Finance and Accounting" as "Theories and Methods of the Multiplication of Factors". The multiplication of factors is the basic formula of the chain substitution method. The core problem is to correctly establish the sequential multiplication sequence of factors, which is related to the accuracy of substitution analysis. There are many theories and methods on how to establish the sequential multiplication sequence, such as: "teleology", "quantity and quality theory", "master-slave relationship theory" and so on. It is believed that the sequence of multiplication of factors should be determined by the essential attributes of economic things. Try to solve the diversity and complexity of the analysis results of different variables in different orders and factors affecting the main factors. Unfortunately, Zhang Xiuqi did not find the reason for the analysis difference from the mathematical mechanism of factor analysis: the interaction between factors. The article questioned the factor analysis method, and failed to find the root cause of the problem and the method to solve the problem. Zhang Zhuzhong (1990) [15] "Discussion on Cost Analysis of Comparable Products" believes that when using this method, attention should be paid to the order of factor substitution. The serial replacement method has no fixed replacement sequence. The sequence should be determined according to the nature of the analyzed factors and the purpose of the analysis. This sequence is recognized by textbooks. But in fact, this accepted analysis principle is often overlooked. In recent years, the source of the order of analysis has been statistical analysis of quantity first and then quality. The article raised the question of alternative order, but did not find the reason for the different order and the different results. Zhou Tieqiu (1988) [16] "Simple formula of difference analysis method" factor analysis method is one of the most important methods in current financial analysis. The so-called factor analysis method is to quantitatively decompose the reasons for changes in a certain comprehensive financial index based on internal comprehensive factors. The article believes that many methods of factor analysis are essentially the form of difference calculation, called the difference analysis method. Wang Zhiqing (1988) [17] "Discussion on Factor Analysis Using the'Dynamic Speed Method"' In order to systematically analyze and study the economic activities of enterprises, the factor method must be used. The correctness of this method directly affects the analysis results. At present, in the teaching and practice of economic activity analysis, the quantitative calculation of factor analysis often uses the chain substitution method (also known as the chain substitution method). This method has different calculation results due to 
different replacement orders, and the replacement basis of each factor is also different, so the degree of influence of the analysis object is also inconsistent, and the more factors, the worse the accuracy. Trying to use a new factor analysis method to solve the long-standing problem of "different order, different results". Luo Shengli (1987) [18] "Discussing factor analysis using dynamic function method" In the analysis of economic activity, several factors change at the same time. When the total number of an analysis object changes, other factors are assumed to be excluded. It is not ruled out that in addition to the influence caused by the changes of some factors, the interaction of the changes of other factors produces the influence, which we call the "public influence". The article found the "public influence" in the product structure model, that is, the "interaction" of the factors, but did not propose a solution.

Cao Jinlin (1986) [20] "A new method of quantitative calculation in factor analysis methods" At present, the quantitative calculation of factor analysis methods often uses the chain substitution subtraction method. Due to the different replacement order, this quantitative calculation method also makes the degree of influence of each factor on the analysis object different. Therefore, in general, this method is not accurate enough and not rigorous enough, and a new quantitative calculation method is proposed. Although the article proposes a proportional method to solve the problem of replacement order, it does not solve the mathematical calculation problem of offsetting positive and negative numbers. She Minglong (1986) [21] "Small discussion on the substitution sequence of the chain substitution method" should systematically analyze and study the economic activities of enterprises, realistically analyze the past, effectively control the present, and scientifically predict the future. Factor analysis methods must be used. The correctness of the method directly affects the analysis result, therefore, it is necessary to study the analysis method. Some opinions on the issue of substitution sequence in linkage substitution methods are discussed. The research conclusion does not help solve the problem of different analysis results caused by the different order of factor analysis. Bie Xingfa (1986) [22] "The Humble Opinions on the Analysis and Calculation Methods of Fund Profit Rate Factors" believes that: my country's fund profit rate factor analysis is calculated through the chain substitution method. The results calculated by this method are not accurate enough and will affect the analysis. It should be changed to a method that combines chain substitution and balanced distribution. The graphic method that attempts to balance the influence of factors in this article is not general.

\subsection{New Method of Factor Analysis, Exponential Logarithmic Ratio Method}

Liu Yun (2019) [32] published an article in "Journal of Finance and Accounting" that factor analysis (also called factor analysis) is an important analysis method in psychology, medicine, economics, statistics, management and accounting. It is also widely used in various fields of social practice. The article analyzes the origin and development history of factor analysis. It can be found from many textbooks and social cases that due to the flaws in the theories and methods of factor analysis and difference analysis, factor analysis has many problems in the dissemination of textbook knowledge and the practical application of society. It is believed that there is no scientific explanation for the difference formed by the interaction between factors. The problems existing in the current factor analysis method are revealed, and the alternative calculation method of the difference is questioned. [33] Based on the status quo and problems of factor analysis, the article uses mathematical geometry and calculus methods to prove by mathematical mechanism that when the factors (or more factors) in the two-factor and three-factor analysis equations are in the product mode, There is an interaction between them; the distribution formula of the interaction is deduced; and the exponential logarithmic conversion method is deduced and tested through calculation. The new factor analysis method can accurately and fairly decompose the interaction between factors. This method is not limited to analyzing the difference between the factors during the reporting period and the base period (actual and budgetary differences); in addition, by analyzing whether the difference between the factors is increasing or decreasing, this is a fair and correct way to decompose the interaction between the factors.. It clarifies the significance of factor analysis and variance analysis, which provides accurate basis for experiment and practice difference analysis and adjustment, and successfully retests and adjusts differences; it also provides a reliable basis for the attribution of difference responsibility and economic management activities. Correct factor analysis and difference analysis not only solve the long-term unsolved problems in economic management; it also solves the problem of the difference between success and failure in comparative experiments. The difference of factors determines the adjustment of the experiment, and the correct analysis of factor difference plays a decisive role in the success of the experiment and has economic significance for improving the results of the experiment. It is revealed from the mathematical mechanism that there is an interaction between factors in the factor difference analysis in the case of the product structure, and the interaction between the factors is not properly apportioned, which is the root cause of the error in the factor analysis and the difference analysis. And deduced the exponential logarithmic ratio calculation formula, which can scientifically solve the problems of factor analysis and variance analysis, and can correctly calculate the influence of factors.

Chen Meihua et al. (2019) [31], the distribution problem of total difference caused by the factor interaction in factor analysis, and the application of a new factor analysis method: exponential logarithmic ratio method, so that factor analysis and difference analysis are correct s solution. This article is mainly based on the factor analysis of the above-mentioned "index logarithmic ratio" method, as well as the current 
American management accounting (Srikant M atar, etc.), cost and management accounting (Charles $\mathrm{T}$ orngren, etc.), and management accounting (Ray $\mathrm{H}$ arrison, etc.). China's current "Applied Statistics" and "Financial Analysis" (national quality courses); "Financial Cost Management" CPA exam book "Cost Management Accounting"; based on the national "Twelfth Five-Year Plan" general higher education undergraduate course "Management Accounting" "As an example, the two calculation methods compare and analyze the calculation results and draw conclusions: the new factor analysis method can correctly allocate factor interactions, and can decompose the main factor differences between factors into factor analysis sums, exponential logarithms The proportional method can solve the problem that cannot be solved by the alternative method of the interaction difference between factors. The exponential logarithmic ratio method solves the unresolved problems that have plagued the factor analysis and difference analysis in economic management for many years. It not only has a theoretical improvement effect, but also has a guiding significance for practical activities. The data of the old and new calculation method cases are derived from the classic works of "Management Accounting" and "Cost Management Accounting" by well-known professors such as the American Accounting Association, Harvard University, Stanford University, etc.; from the Chinese CPA Examination "Financial Cost Management" writing group, the Chinese people The writings of famous professors such as universities, Central University of Finance and Economics, Xi'an Jiaotong University are representative. The writings of other famous professors are representative.

"Factor Difference Analysis and Research in Abnormal Conditions of Indicators" follows the problems of factor analysis and difference analysis, the interaction index logarithmic ratio method, and the comparison of the calculation results of the new and old calculation methods of factor analysis. Re-analysis and research under abnormal conditions of indicators in quantitative analysis. It omits the background, current situation, and significance of the proposition, omits the problem, the cause of the problem, the method of solving the problem, the application of the case, the comparison of the new and the old methods, etc. (see References [31-33] for details). Or is it a supplement and improvement to the previous article. The indicator abnormality here mainly refers to the factors analyzed. The reporting period (actual number) is positive, and the base period (or budgeted number, standard number) is negative; the reporting period (actual number) is negative, and the base period (or budgeted number, standard number) is Positive number; the reporting period (actual number) is a negative number, and the base period (or budget number, standard number) is also negative, but the reporting period is greater than the base period; the reporting period (actual number) is a negative number, and the base period (or budget number, standard number) is also It is a negative number, but the number of reporting periods is less than the number of base periods. It is solved by the logarithmic ratio method of the absolute value of the difference, so that the factor analysis and the difference analysis refer to the logarithmic ratio method, which can be applied regardless of the abnormal situation of the index.

In order to be more realistic, in the case analysis of listed companies, we found the factor analysis conditions in the complex situation with abnormal data that meets the proposition requirements, that is, the comprehensive analysis data of the performance factors of ZXGS company from 2016 to 2019, which is satisfied from the technical level. The complexity of the problem to be solved is suitable for the case.

\section{The Basic Equation of Factor Variance Analysis}

\subsection{The Basic Equation of Factor Analysis}

An abnormal indicator means that the numerator and denominator of the compared factor index are inconsistent with the logarithm, or the numerator and denominator are both negative (the principle of negative and positive in mathematics is contrary to the actual meaning of economics), and the current year cannot be used. The ratio to the previous year represents the change in the difference.

Data source case background: ZXGS listed company, due to technology patents, was punished by the technology patent country with a penalty of US\$2.29 billion in 2016, and its performance was greatly affected. After the company was hit hard, its economic benefits are now gradually recovering. From 2016 to 2019, ZXGS's operating performance was ups and downs. In the past four years, the economic indicators that reflect performance are like riding a cable car, reaching a trough in one year; and rising to a peak in one year. This ups and downs are manifested in accounting indicators, either positive or negative, or high or low; it satisfies the needs of factor analysis and the different situations of the abnormal (variable) instances of factor indicators, and conforms to the event analysis procedure: factor analysis, variance Analysis and characteristics of financial indicators. Event analysis is an unknown event in advance under normal circumstances, and the event is confirmed after analysis; in this example, it is an analysis of known events, because it satisfies the factor analysis and variance analysis conditions under abnormal indicators.

Take the examples of the increase and decrease of the return on net assets of ZXGS in recent years to explain the complex cases of factor analysis and variance analysis, so that the factor analysis index logarithmic ratio method is more perfect, and the theory and practice are better integrated.

The basic equation of analysis refers to the correlation equation in the following cases. It is a mixed structure model. Of course, it can also be other structure models, but it must have a factor product structure. In order to facilitate comprehensive analysis, we take the return on net assets as the main factor, and use the factor expansion method (the expansion process is omitted) to decompose into several 
factors: return on total assets, debt leverage coefficient, debt capital cost rate, net interest rate after tax (1-Income tax rate) The basic equation of factor analysis is a mixed structure, that is, a structural model coexisting with a structure and a product structure. In their fractional structure, there is an interaction between the factors of the factors and the main factors.

Use ROE to denote the return on net assets, $\mathrm{k}_{1}$ denotes the return on total assets, $\mathrm{k}_{2}$ denotes the debt leverage coefficient, $\mathrm{k}_{3}$ table (1-income rate), $\mathrm{k}_{4}$ denotes the debt capital cost rate, and the factorization in the formula is:

$$
R 0 E=\left[k_{1}+k_{2}\left(k_{1}-k_{4}\right)\right] \times k_{3} \rightarrow\left\{\begin{array}{c}
k_{1} \cdot k_{3} \\
k_{2} \cdot k_{1} \cdot k_{3} \\
-k_{2} \cdot k_{1} \cdot k_{4}
\end{array}\right.
$$

Formula (1) divides the main factors into three groups, each group is a product factor, in each factor, the influence of the factor on the main factor is the interaction between the factors, and then the factor is decomposed by the exponential logarithmic ratio method. Other mixed structure models can also be used as basic equations for factor analysis and variance analysis.

\subsection{Financial Indicator Data Source}

Table 1. ZXGS's main financial indicators in the past five years.

\begin{tabular}{lllllll}
\hline Period & Assets & Debt & TP & IT & FE & NP \\
\hline $2019 / 12 / 31$ & $1.41 \mathrm{E}+11$ & $1.03 \mathrm{E}+11$ & $7.16 \mathrm{E}+09$ & $1.39 \mathrm{E}+09$ & $9.66 \mathrm{E}+08$ & $3.8 \mathrm{E}+10$ \\
$2018 / 12 / 31$ & $1.29 \mathrm{E}+11$ & $9.64 \mathrm{E}+10$ & $-7.4 \mathrm{E}+09$ & $-4 \mathrm{E}+08$ & $2.81 \mathrm{E}+08$ & $3.3 \mathrm{E}+10$ \\
$2017 / 12 / 31$ & $1.44 \mathrm{E}+11$ & $9.86 \mathrm{E}+10$ & $6.72 \mathrm{E}+09$ & $1.33 \mathrm{E}+09$ & $1.04 \mathrm{E}+09$ & $4.54 \mathrm{E}+10$ \\
$2016 / 12 / 31$ & $1.42 \mathrm{E}+11$ & $1.01 \mathrm{E}+11$ & $-7.7 \mathrm{E}+08$ & $6.4 \mathrm{E}+08$ & $2.08 \mathrm{E}+09$ & $5.39 \mathrm{E}+09$ \\
\hline
\end{tabular}

The total profit: TP, Income tax: IT, Financial expenses: FE, Net assets: NA, Net profit: NP, Same below.

Convert the financial data in Table 1 into equation element indicators. In the calculation of the relative number of daily accounting indicators, the calculation of the return on net assets is usually, the net profit of the molecule is a dynamic indicator, which is an indicator of the company during a certain period of operation; convert the static indicator of net assets As a dynamic indicator, the numerator and denominator, the dynamic ratio is often used (beginning net assets + ending net assets) $/ 2$ method to transform into dynamic financial indicators, which are regarded as dynamic financial indicators for the period, which are comparable. In this example, the dynamic and static properties of the index are not considered, and all the static cross-sectional data at the time point are used to directly calculate the relative number index.

Table 2. Converted into equation element indicators.

\begin{tabular}{lllllll}
\hline Period & ROE & EBIT & ROA & DCC & DL & 1-ITR \\
\hline $2019 / 12 / 31$ & $15.22 \%$ & $8.13 \mathrm{E}+09$ & $5.76 \%$ & $0.94 \%$ & 2.72 & $80.66 \%$ \\
$2018 / 12 / 31$ & $-21.08 \%$ & $-7.07 \mathrm{E}+09$ & $-5.47 \%$ & $0.29 \%$ & 2.92 & $94.55 \%$ \\
$2017 / 12 / 31$ & $11.87 \%$ & $7.76 \mathrm{E}+09$ & $5.39 \%$ & $1.06 \%$ & 2.17 & $-21.08 \%$ \\
$2016 / 12 / 31$ & $-3.44 \%$ & $-5.60 \mathrm{E}+08$ & $-0.40 \%$ & $0.21 \%$ & 2.46 & $11.87 \%$ \\
\hline
\end{tabular}

Debt capital cost: DCC, Debt leverage: DL, 1- income tax rate: 1-ITR, Same below.

Calculation tool: Excel2019 version.

\section{Factor Analysis Variance Analysis}

For the convenience of research, we use a to represent the reporting period index, $\mathrm{a}_{0}$ to represent the base period index, $\Delta \mathrm{a}$ to represent the variation difference, $\Delta \mathrm{a} / \mathrm{a}_{0}$ to represent the variation range, and ln to represent the natural logarithm (the logarithm just serves as a bridge here. Any natural number greater than 1 as the base logarithm can be used). Taking into account the length of the article, the calculation formula of the exponential logarithmic ratio method will not be repeated in this article. For details, please refer to Reference [33].

\subsection{The Reporting Period Is Positive and the Base Period Is Negative}

The return on total assets for the reporting period is $5.76 \%$ in 2019, and the base period is $-5.47 \%$ in 2018. Change difference: $\Delta \mathrm{a}=\mathrm{a}_{1}-\mathrm{a}_{0}=11.22 \%$, change ratio logarithm $\ln (11.22 \% /|-5.47 \%|)=0.7194$, overall logarithmic weight is 0.56055 , instead of fractional combination product logarithm $\ln (0.04643 /|-0.0517|)$. The combination of factors in the following table is mainly based on the apportionment of interaction between the factors in the continuous product mode difference analysis.

Table 3. Comprehensive analysis of the difference between ZXGS company's return on net assets in 2019 and 2018.

\begin{tabular}{|c|c|c|c|c|c|}
\hline FactorCombination & 2019 year & 2018 year & Difference & Logarithm change & Factor influence \\
\hline $\mathrm{ROA}$ & 0.0576 & -0.0547 & 0.1122 & 0.7194 & 0.1259 \\
\hline 1-ITR & 0.8066 & 0.9455 & -0.1389 & -0.1588 & -0.0278 \\
\hline product & 0.0464 & -0.0517 & 0.0981 & 0.5606 & 0.0981 \\
\hline
\end{tabular}




\begin{tabular}{|c|c|c|c|c|c|}
\hline FactorCombination & 2019 year & 2018 year & Difference & Logarithm change & Factor influence \\
\hline ROA & 0.0576 & -0.0547 & 0.1122 & 0.7194 & 0.4088 \\
\hline DL & 2.7203 & 2.9244 & -0.2041 & -0.0723 & -0.0411 \\
\hline 1-ITR & 0.8066 & 0.9455 & -0.1389 & -0.1588 & -0.0903 \\
\hline product & 0.1263 & -0.1511 & 0.2774 & 0.4882 & 0.2774 \\
\hline DL & 2.7203 & 2.9244 & -0.2041 & -0.0723 & -0.0010 \\
\hline DCC & 0.0094 & 0.0029 & 0.0064 & 1.1673 & 0.0156 \\
\hline 1-ITR & 0.8066 & 0.9455 & -0.1389 & -0.1588 & -0.0021 \\
\hline product & 0.0205 & 0.0081 & 0.0125 & 0.9361 & 0.0125 \\
\hline Check & 0.1522 & -0.2108 & 0.3630 & & 0.3630 \\
\hline
\end{tabular}

Table 4. The impact of four factors on return on net assets.

\begin{tabular}{llllll}
\hline & ROA & 1-ITR & DL & DCC & 工 \\
\hline FIL & $53.47 \%$ & $-11.59 \%$ & -0.04 & $-1.56 \%$ & $36.30 \%$ \\
\hline
\end{tabular}

Four-factor influence: FIL. Same below.

That is, the return on net assets in 2019 increased by $36.3 \%$ compared to 2018 , because the return on total assets increased by $53.47 \%$, the income tax factor decreased by $11.59 \%$, the debt leverage factor decreased by 0.04 , and the debt capital cost ratio decreased by $1.56 \%$.

\subsection{The Reporting Period Is Negative and the Base Period Is Positive}

Still taking the ZXGS company as an example, the hybrid model of return on net assets is divided into three factor combinations, and there is an interaction between them. The return on total assets index is negative in 2018 and positive in 2017. The logarithm of change is used to solve the interaction apportionment in the case of variation. The difference of the change is: $\Delta \mathrm{a}=\mathrm{a}_{1}-\mathrm{a}_{0}=-10.86 \%$, then the logarithm of the change is $-\ln (|-10.86 \%| / 5.39 \%)=-0.6999$, and the sum of its weights cannot be changed by the product of the factor combination The logarithm of the exponent is the sum of the logarithm of the change ratio of each factor.

Table 5. Analysis of the difference between the factors of ZXGS's return on net assets in 2018 and 2017.

\begin{tabular}{|c|c|c|c|c|c|}
\hline FactorCombination & 2019 year & 2018 year & Difference & Logarithm change & Factor influence \\
\hline $\mathrm{ROA}$ & -0.0547 & 0.0539 & -0.1086 & -0.6999 & -0.1242 \\
\hline 1-ITR & 0.9455 & 0.8017 & 0.1438 & 0.1650 & 0.0293 \\
\hline product & -0.0517 & 0.0432 & -0.0949 & -0.5350 & -0.0949 \\
\hline DL & 2.9244 & 2.1724 & 0.7520 & 0.2973 & 0.3064 \\
\hline 1-ITR & 0.9455 & 0.8017 & 0.1438 & 0.1650 & 0.1701 \\
\hline product & -0.1511 & 0.0939 & -0.2450 & -0.2377 & -0.2450 \\
\hline DL & 2.9244 & 2.1724 & 0.7520 & 0.2973 & 0.0037 \\
\hline DCC & 0.0029 & 0.0106 & -0.0077 & -1.2907 & -0.0162 \\
\hline 1-ITR & 0.9455 & 0.8017 & 0.1438 & 0.1650 & 0.0021 \\
\hline product & 0.0081 & 0.0184 & -0.0104 & -0.8285 & -0.0104 \\
\hline Check & -0.2108 & 0.1187 & -0.3295 & & -0.3295 \\
\hline
\end{tabular}

The impact of four factors on the rate of return on net assets:

The return on net assets decreased by $32.95 \%$ in 2018 compared to 2017 . This was because the decline in the return on total assets affected $84.57 \%$, the income tax affected $19.73 \%$, the leverage factor affected 0.3 , and the cost of debt funds affected $1.62 \%$.

Table 6. Analysis results of the difference in return on net assets between 2018 and 2017.

\begin{tabular}{llllll}
\hline & ROA & 1-ITR & DL & DCC & $\boldsymbol{\Sigma}$ \\
\hline FIL & $-84.57 \%$ & $19.73 \%$ & $30.27 \%$ & $1.62 \%$ & $-32.95 \%$ \\
\hline
\end{tabular}

\subsection{The Reporting Period Is the Same as the Base Period, and the Reporting Period Is Greater Than the Base Period}

In factor analysis, the reporting period and the base period can be called the analysis period and the standard period respectively. Usually, the object to be analyzed is called the analysis period, that is, the analysis period; what period indicator is used as the comparison standard is called the standard period. The data may not be in a continuous period. In order to solve the comparability of the replication situation in different periods, we have selected the analysis period and the standard period. Still using ZTE data as an example, we use 2016 indicators as the analysis period and 2018 as the standard period, that is, using indicators such as return on net assets in 2018 as the standard to analyze the changes in indicators from 2016 to 2018. The analysis criteria are the divisors and subtracts in mathematics; the indicators in the analysis period are the dividends and subtracts. The intertemporal comparison is because the indicators between them satisfy the complexity encountered in social practice.

The return on total assets satisfies the propositional conditions: $\mathrm{a}_{1}<0, \mathrm{a}_{0}<0$ are all negative numbers, and $\mathrm{a}_{1}>\mathrm{a}_{0}$ change difference $\Delta \mathrm{a}=\mathrm{a}_{1}-\mathrm{a}_{0}=0.0507$, and the logarithm of 
change is $\ln (0.0507 /|-0.0546|)=0.0751$, the logarithm of the exponent of the combined product cannot be used as the sum of the combined weights, and the sum of the logarithms of each factor is used as the weight.

Table 7. Analysis of the factors of ZXGS company's 2016-to-2018 return on net assets.

\begin{tabular}{|c|c|c|c|c|c|}
\hline FactorCombination & 2019 year & 2018 year & Difference & Logarithm change & Factor influence \\
\hline ROA & -0.0040 & -0.0547 & 0.0507 & 0.0751 & 0.0045 \\
\hline 1-ITR & 1.8338 & 0.9455 & 0.8883 & 0.6624 & 0.0399 \\
\hline product & -0.0072 & -0.0517 & 0.0444 & 0.7375 & 0.0444 \\
\hline ROA & -0.0040 & -0.0547 & 0.0507 & 0.0751 & 0.0177 \\
\hline DL & 2.4644 & 2.9244 & -0.4600 & -0.1712 & -0.0403 \\
\hline 1-ITR & 1.8338 & 0.9455 & 0.8883 & 0.6624 & 0.1558 \\
\hline product & -0.0179 & -0.1511 & 0.1332 & 0.5664 & 0.1332 \\
\hline $\mathrm{DL}$ & 2.4644 & 2.9244 & -0.4600 & -0.1712 & -0.0015 \\
\hline DCC & 0.0021 & 0.0029 & -0.0008 & -0.3450 & -0.0030 \\
\hline 1-ITR & 1.8338 & 0.9455 & 0.8883 & 0.6624 & 0.0057 \\
\hline product & 0.0093 & 0.0081 & 0.0013 & 0.1463 & 0.0013 \\
\hline Check & -0.0344 & -0.2108 & 0.1764 & & 0.1764 \\
\hline
\end{tabular}

Table 8. The analysis results of the difference in the return on net assets of ZXGS between 2016 and 2018.

\begin{tabular}{|c|c|c|c|c|c|}
\hline & ROA & 1-ITR & DL & DCC & $\Sigma$ \\
\hline FIL & $2.22 \%$ & $19.00 \%$ & $-3.88 \%$ & $0.30 \%$ & $17.64 \%$ \\
\hline
\end{tabular}

The return on net assets in 2016 increased by $17.64 \%$ compared to 2018 , which is due to the increase in the return on total assets by $2.22 \%$, the increase in income tax by $19 \%$, the decrease in debt leverage by $4 \%$, and the $0.3 \%$ impact on the cost of debt.

\subsection{The Reporting Period Is the Same as the Base Period, and the Reporting Period Is Less than the Base Period}

In the above example, 2018 is used as the analysis period, and the 2016 indicator is used as the analysis standard, that is, the above case data is reversed. The return on total assets indicator satisfies: $\mathrm{a}_{1}<0, \mathrm{a}_{0}<0$ are all negative numbers, and $\mathrm{a}_{1}<\mathrm{a}_{0}$, change difference $\Delta \mathrm{a}=\mathrm{a}_{1}-\mathrm{a}_{0}<0$, change ratio $\operatorname{logarithm}-\ln \left(\left|\mathrm{a}_{1}-\mathrm{a}_{0}\right| /\left|\mathrm{a}_{0}\right|\right)$; the sum of logarithmic weights, the sum of the logarithms of changes by each factor.

Table 9. Analysis of the difference between ZXGS's 2018 and 2016 net asset return factors.

\begin{tabular}{|c|c|c|c|c|c|}
\hline FactorCombination & 2019 year & 2018 year & Difference & Logarithm change & Factor influence \\
\hline $\mathrm{ROA}$ & -0.0547 & -0.0040 & -0.0507 & -2.5513 & -0.0353 \\
\hline 1-ITR & 0.9455 & 1.8338 & -0.8883 & -0.6624 & -0.0092 \\
\hline product & -0.0517 & -0.0072 & -0.0444 & -3.2138 & -0.0444 \\
\hline ROA & -0.0547 & -0.0040 & -0.0507 & -2.5513 & -0.1117 \\
\hline DL & 2.9244 & 2.4644 & 0.4600 & 0.1712 & 0.0075 \\
\hline 1-ITR & 0.9455 & 1.8338 & -0.8883 & -0.6624 & -0.0290 \\
\hline product & -0.1511 & -0.0179 & -0.1332 & -3.0426 & -0.1332 \\
\hline $\mathrm{DCC}$ & 0.0029 & 0.0021 & 0.0008 & 0.3450 & 0.0030 \\
\hline 1-ITR & 0.9455 & 1.8338 & -0.8883 & -0.6624 & -0.0057 \\
\hline product & 0.0081 & 0.0093 & -0.0013 & -0.1463 & -0.0013 \\
\hline Check & -0.2108 & -0.0344 & -0.1764 & & -0.1764 \\
\hline
\end{tabular}

The return on net assets in 2018 compared with 2016, decreased by $17.64 \%$ (in line with the above increase), because the return on total assets reduced the main factor by $14.7 \%$, the income tax reduced the main factor by $3.24 \%$, and the debt leverage caused the main factor. Increased by $1 \%$, the debt capital cost ratio reduces the main factor by $3 \%$.

Table 10. ZXGS company's 2018 and 2016 return on net assets factor difference analysis results.

\begin{tabular}{llllll}
\hline & ROA & 1-ITR & DL & DCC & $\boldsymbol{\Sigma}$ \\
\hline FIL & $-14.70 \%$ & $-3.24 \%$ & $0.6 \%$ & $-0.30 \%$ & $-17.64 \%$ \\
\hline
\end{tabular}

The factor influence is not the opposite value of the above data. The main reason is that the ratio standard has changed, but the total decrease rate is consistent with the increase rate data.
Suppose we don't know why ZXGS company is in the analysis period. What causes the abnormal return on net assets index and factor indicators. Through the above: operating profit \pm non-operating income and expenditure total profit-income tax net profit analysis chain found, 2018 In the March quarterly report, non-operating expenses were 6.732 billion yuan, and at the end of 2016, non-operating expenses were 6.295 billion yuan, with a total expenditure of 13.07 billion yuan (RMB), resulting in abnormal company profit indicators. The company's profit index is abnormal, and it is reversed: a major incident has occurred in the company, and the major incident has affected the company's performance. Because the "indicator abnormal text" only considers technical method issues, not the application of technical method results, and ignores the reasons for the analysis results, item evaluations, management suggestions, etc. 


\section{Analysis Conclusion}

Based on the above analysis, we have solved the problems in the comprehensive analysis of the product structure and the factor analysis of various conditions of indicator variation. The solution is to use the exponential logarithmic ratio method to describe the difference between the reporting period and the base period, and appropriately take the logarithm of the degree of change, that is, $\ln \left(\Delta \mathrm{a} / \mathrm{a}_{0}\right)$, and first determine whether the population is rising or falling; then according to the positive ratio In the case of negative sign, take the absolute value of the negative sign so that the ratio can be logarithm; finally, the overall difference is decomposed according to the weight of the sum of the exponential logarithm (without variation) of the ratio logarithm. In this way, the interaction effect on the overall impact in the complex multi-factor product structure is scientifically and reasonably decomposed into each factor.

Theoretical basis: exponential logarithmic ratio method, basic theory: the reporting period a1 and the base period a0 are both positive, that is, $a_{1}>0, a_{0}>0$, use $\ln \left(a_{1} / a_{0}\right)$ to represent the degree of change in different periods, when $a_{1}>a_{0}$ is When the value of the logarithm is positive, the reporting period is greater than the base period; when $a_{1}<a_{0}$, the logarithm is negative, and the reporting period is lower than the base period. What we are encountering now is that the amount of the reporting period and the base period are different signs, that is, one positive and one negative. According to the mathematical understanding, the value of the numerator and the denominator is negative, and the logarithm cannot be taken; and when the numerator and denominator are both It is a negative number $\mathrm{a}_{1}<0, \mathrm{a}_{0}<0$, there are two cases $a_{1}>a_{0}, a_{1}<a_{0}$, does this mean that the "exponential logarithm method" cannot be solved. In fact, this is not the case. We use the principle of exponential logarithmic ratio method to extend the "variation difference ratio logarithmic ratio method". These practical cases can also be solved. We are talking about a comprehensive analysis of multi-factor combinations. Single indicators will also encounter these problems. For example, we often see the net profit growth indicator column in the financial analysis growth ability analysis on the Internet. The column of continuous loss cannot be filled. In the column of return on net assets, the net profit of the current period is a negative number at a loss, the company is already insolvent, and the net assets are also negative. It is impossible to fill in the table with a negative to be positive. Only use "- -" The symbol indicates that, in fact, many of these complicated situations can also be solved by the absolute value difference logarithmic ratio method (the case is omitted).

Considering the different signs in the numerator and denominator, there are four cases in which the same negative signs are large and small. Taking two factors as an example, suppose that the data of the $b$-factor indicator reporting period and the base period are normal, both are positive, and $\mathrm{b}_{1}>0, \mathrm{~b}_{0}>0$, the main factor is $\mathrm{F}$, the basic equation of analysis is $\mathrm{F}=\mathrm{a} \cdot \mathrm{b}$, and the difference of factor analysis is
$\Delta \mathrm{F}=\mathrm{a}_{1} \cdot \mathrm{b}_{1}-\mathrm{a}_{0} \cdot \mathrm{b}_{0}$. The research conclusions are as follows:

1) When the numerator is positive and the denominator is negative, $a_{1}>0, a_{0}<0$, we take the logarithm of the variation, the variation is $\ln \left(\left(\Delta a=a_{1}-a_{0}\right) /\left|a_{0}\right|\right)$, so the numerator is positive Number, the denominator is also positive, you can take the logarithm, the calculation result a factor is increased. What needs to be added is the sum of the logarithmic proportions of the overall difference, and the sum of the logarithms of each factor needs to be added, that is, the logarithm of the ratio of the change difference to the absolute value of the base period is used for the proportional weight.

a Factor influence:

$$
\frac{\ln \left(\left(a_{1}-a_{0}\right) /\left|a_{0}\right|\right)}{\ln \left(\left(a_{1}-a_{0}\right) /\left|a_{0}\right|\right)+\ln \left(\left(b_{1}-b_{0}\right) / b_{0}\right)} \cdot\left(\mathrm{a}_{1} \cdot \mathrm{b}_{1}-\mathrm{a}_{0} \cdot \mathrm{b}_{0}\right)
$$

b Factor influence:

$$
\frac{\ln \left(\left(b_{1}-b_{0}\right) / b_{0}\right)}{\ln \left(\left(a_{1}-a_{0}\right) /\left|a_{0}\right|\right)+\ln \left(\left(b_{1}-b_{0}\right) / b_{0}\right)} \cdot\left(\mathrm{a}_{1} \cdot \mathrm{b}_{1}-\mathrm{a}_{0} \cdot \mathrm{b}_{0}\right)
$$

2) When the numerator is negative and the denominator is positive, $a_{1}<0, a_{0}>0$, we take the logarithm of the ratio of the absolute value of the negative change range to the absolute value of the base period, namely $-\ln \left(\left(\left|a_{1}-a_{0}\right|\right) / a_{0}\right)$; The main factor cannot use the logarithm of the total difference, the sum of the total logarithm, and use the logarithm of the variation range of each factor to add. Taking two factors as an example, suppose that the data of the index reporting period and the base period of factor $b$ are both positive, and $\mathrm{b}_{1}>\mathrm{b}_{0}$, the main factor is $\mathrm{F}$, the basic equation of analysis is $\mathrm{F}=\mathrm{a} \cdot \mathrm{b}$, and the difference of factor analysis is $\Delta \mathrm{F}=\mathrm{a}_{1} \cdot \mathrm{b}_{1}-\mathrm{a}_{0} \cdot \mathrm{b}_{0}$ a Factor influence:

$$
\frac{-\ln \left(\left(\left|a_{1}-a_{0}\right|\right) / a_{0}\right)}{-\ln \left(\left(\left|a_{1}-a_{0}\right|\right) / a_{0}\right)+\ln \left(\left(b_{1}-b_{0}\right) / b_{0}\right)} \cdot\left(\mathrm{a}_{1} \cdot \mathrm{b}_{1}-\mathrm{a}_{0} \cdot \mathrm{b}_{0}\right)
$$

b Factor influence:

$$
\frac{\ln \left(\left(b_{1}-b_{0}\right) / b_{0}\right)}{-\ln \left(\left(\left|a_{1}-a_{0}\right|\right) / a_{0}\right)+\ln \left(\left(b_{1}-b_{0}\right) / b_{0}\right)} \cdot\left(\mathrm{a}_{1} \cdot \mathrm{b}_{1}-\mathrm{a}_{0} \cdot \mathrm{b}_{0}\right)
$$

3) When the numerator and denominator are both negative and the reporting period is greater than the base period, that is, when $\mathrm{a}_{1}<0, \mathrm{a}_{0}<0$ and $\mathrm{a}_{1}>\mathrm{a}_{0}$, the change difference $\Delta \mathrm{a}=\mathrm{a}_{1}-\mathrm{a}_{0}>0$, the logarithm of the change $\ln \left(\Delta \mathrm{a} /\left|\mathrm{a}_{0}\right|\right.$, the sum of the logarithmic weights cannot be expressed by the logarithm of the overall change range, and the value of the logarithm of each factor is added. Taking two factors as an example, assume that the data of the b-factor indicator during the reporting period and the base period are both positive, and $b_{1}>b_{0}$, the main factor is $F$, the basic equation of analysis is $\mathrm{F}=\mathrm{a} \cdot \mathrm{b}$, the difference of factor analysis is $\Delta \mathrm{F}=\mathrm{a}_{1} \cdot \mathrm{b}_{1}-\mathrm{a}_{0} \cdot \mathrm{b}_{0}$

a Factor influence:

$$
\frac{\ln \left(\left(a_{1}-a_{0}\right) /\left|a_{0}\right|\right)}{\ln \left(\left(a_{1}-a_{0}\right) /\left|a_{0}\right|\right)+\ln \left(\left(b_{1}-b_{0}\right) / b_{0}\right)} \cdot\left(\mathrm{a}_{1} \cdot \mathrm{b}_{1}-\mathrm{a}_{0} \cdot \mathrm{b}_{0}\right)
$$

b Factor influence:

$$
\frac{\ln \left(\left(b_{1}-b_{0}\right) / b_{0}\right)}{\ln \left(\left(a_{1}-a_{0}\right) /\left|a_{0}\right|\right)+\ln \left(\left(b_{1}-b_{0}\right) / b_{0}\right)} \cdot\left(\mathrm{a}_{1} \cdot \mathrm{b}_{1}-\mathrm{a}_{0} \cdot \mathrm{b}_{0}\right)
$$


4) When the numerator and denominator are both negative and the reporting period is less than the base period, that is, when $\mathrm{a}_{1}<0, \mathrm{a}_{0}<0$ and $\mathrm{a}_{1}<\mathrm{a}_{0}$, the change difference $\Delta \mathrm{a}=\mathrm{a}_{1}-\mathrm{a}_{0}<0$, the logarithm of the change range $-\ln \left(|\Delta \mathrm{a}| /\left|\mathrm{a}_{0}\right|\right)$, the sum of the logarithmic weights cannot be expressed by the logarithm of the overall variation range, and the logarithmic values of each factor are added together.

a Factor influence:

$$
\frac{-\ln \left(\left(\left|a_{1}-a_{0}\right|\right) /\left|a_{0}\right|\right)}{-\ln \left(\left(\left|a_{1}-a_{0}\right|\right) /\left|a_{0}\right|\right)+\ln \left(\left(b_{1}-b_{0}\right) / b_{0}\right)} \cdot\left(\mathrm{a}_{1} \cdot \mathrm{b}_{1}-\mathrm{a}_{0} \cdot \mathrm{b}_{0}\right)
$$

b Factor influence:

$$
\frac{\ln \left(\left(b_{1}-b_{0}\right) / b_{0}\right)}{-\ln \left(\left(\left|a_{1}-a_{0}\right|\right) /\left|a_{0}\right|\right)+\ln \left(\left(b_{1}-b_{0}\right) / b_{0}\right)} \cdot\left(\mathrm{a}_{1} \cdot \mathrm{b}_{1}-\mathrm{a}_{0} \cdot \mathrm{b}_{0}\right)
$$

We use the exponential logarithmic ratio method to solve the problem of factor interaction apportionment in the product structure model. The key method is to introduce the $\ln \left(\mathrm{a}_{1} / \mathrm{a}_{0}\right)$ ratio; in complex situations, the factor interaction when the reporting period indicators are inconsistent with the base period indicators For function apportionment, we introduce the key calculation method of $\ln \left(\Delta \mathrm{a} / \mathrm{a}_{0}\right)$. When the influence is reduced, the negative sign is added before the logarithm value; when the numerator and denominator of the logarithmic ratio method of the degree of change is different, the negative sign is added to the absolute value. The amount of influence can be increased or decreased; when the sign is both negative at that time, according to the above conditions 3 and 4, the absolute value difference ratio is weighted against the value. If the b-factor index is also abnormal, the above method is still applicable.

After we have solved the factor analysis in the above complex situations, compared with the "index logarithmic ratio" method, named the "variation ratio logarithmic ratio" method, single indicator calculation growth ability analysis, such as net profit growth rate (reporting period and The base period is all negative), calculated by the change ratio plus the absolute value logarithm method, in the web page, do not use the "--" symbol to indicate it, this may be another contribution of this article. Some people may think that this kind of calculation is very troublesome and inconvenient to popularize and apply; in fact, it is not the case, we only need to add the conditional selection formula IF function in the Excel table module.

This article is the follow-up research results of the factor analysis and the difference analysis index logarithmic ratio method. The previous research results of related topics can be found in the references of this article [31-33]; this article solves the abnormal factor difference between the reporting period and the base period. Quantity analysis can be named: the logarithmic ratio method of the ratio of the absolute value of the difference to the absolute value of the base period. This constitutes a complete factor analysis system with factor analysis and variance analysis index logarithmic ratio method. In the case of abnormal indicators described in the case, the "abnormal" here mainly refers to situations that are rare in textbooks but do exist in social practice. The logarithmic ratio method of the ratio of the absolute value of the range of change to the absolute value of the base period is adopted for the abnormal situation of the indicator, so that we can correctly analyze the factors and decompose the difference, and make the index logarithm regardless of whether the indicator is positive or negative. The proportional method is more complete, scientific and correct; the factor analysis and the difference analysis index logarithmic ratio method are completely dedicated to the author, which not only enriches and perfects the accounting method, but also confirms the natural attributes of the connection between accounting and its methods and mathematics There is a natural connection between accounting and mathematics, which shows that accounting has natural attributes; accounting systems and subject settings are social attributes of accounting; accounting is a subject with both natural and social attributes.

Research conclusion: no matter how abnormal the index of factor analysis and difference analysis is, we can use the exponential logarithmic scale method to solve the problem; However, in the case of abnormal indicators, the logarithmic scale method of variable ratio plus absolute value is used. It is slightly different from the exponential-logarithmic proportional method under the normal condition of indicators. Complete factor analysis, difference analysis, to correct the current Chinese and foreign "Management Accounting", "Financial Management", "Financial Analysis", "Financial Statement Analysis", "Cost Management Accounting", "Financial Cost Management", "Cost Accounting" and other textbooks related knowledge errors, to provide a basis; To fairly distinguish the responsibility of the subject of economic activities, provide quantitative basis; In order to improve the probability of success of physics, chemistry and medical experiment, provide accurate difference analysis and correction basis. This is the contribution of this article.

\section{References}

[1] Project Funding: Factor Analysis and Dupont Analysis Improvement, code: 2018WQNCX310, Big Data and Accounting, Internal audit approach: 2016GXJK227.

[2] Roman L. Weil (Roman L. Weil), Katherine Schipper (Katherine Schipper) and Jennifer Francis (Jennifer Francis), translated by $\mathrm{Zhu}$ Dan and $\mathrm{Qu}$ Tenglong. Financial Accounting-Concepts, Methods and Applications ("Financial Accounting" "Fourteenth edition of the original book) [M]. Machinery Industry Press, September 2015, the first edition P 175-176.

[3] Srikant M. Datar (Srikant M. Datar) Madhav V. Rajan (Madhav V. Rajan) "Management Accounting" [M]. (Translated by Wang Liyan, Chen Jiaxi, etc., China Renmin University Press, April 2015, the first edition) Chapter 13 "Flexible Budget, Difference and Management Control" p 461-463.

[4] Zhang Xianzhi, Chen Youbang, "Financial Analysis" (8th edition) [M]. "Twelfth Five-Year" general higher education undergraduate national planning textbook, national quality courses, Dongbei University of Finance and Economics Press, March 2017 edition P170. 
[5] Charles T. Horngren Srikant M. Datar Madhav V. Rajan Wang Liyan and Liu Yingwen translated "Cost and Management Accounting" (15th edition) [M]. China Renmin University Press, June 2016, first edition, 2017 P464-470 was printed for the third time in November.

[6] Organized and compiled by the Chinese Institute of Certified Public Accountants, CPA exam book 2020 CPA National Unified Examination Guidance Textbook "Financial Cost Management" [M]. China Finance Publishing Media Group, China Finance and Economic Publishing House, March 2020 edition P35-41.

[7] "Cost Management Accounting" by Meng Yan and Liu Junyong $[\mathrm{M}]$. National planning textbook for undergraduates during the Twelfth Five-Year Plan for general higher education, printed by Higher Education Press for the 3rd time in December 2017, P109.

[8] "Corporate Financial Analysis" edited by Wu Xiaoling and Tian Gaoliang [M]. 21st Century Economics and Management Planning Textbook, 2nd print by Peking University Press in February 2018, P17-19.

[9] Wen Subin "Management Accounting: Theory·Model-Case" [M]. Excellent planning textbook, Mechanical Industry Press, June 2014 P231.

[10] Sun Maozhu, Zhi Xiaoqiang, Dai Lu, editor in chief, National Outstanding Achievement Award, "Management Accounting" (8th edition), a national undergraduate planning textbook for general higher education in the "Twelfth Five-Year" period [M] Published by Renmin University of China Society P215-216.

[11] Peng Jiaqing. Application example analysis of factor analysis method in coal mine production cost analysis [J]. Friends of Accounting (first issue), 2008, (07): 60-61.

[12] Zhang Daoyun. The origin of the formula in the factor analysis of the task completion of the cost reduction of comparable products $[\mathrm{J}]$. National Business Situation (Economic Theory Research), 2008, (07): 35-37.

[13] Li Shanzhai. Discussion on the unified formula in factor analysis [J]. Journal of Jimei University (Natural Science Edition), 1997, (03): 6-10.

[14] Zhang Xiuqi. The theory and method of factor multiplication [J]. Finance and Accounting, 1991, (07): 49-50.

[15] Zhang Zhuzhong. Discussion on the Cost Analysis of Comparable Products [J]. Accounting Research, 1990 (01): $39-45$.

[16] Zhou Tieqiu. Simple formula of difference analysis method [J]. Finance and Accounting, 1988 (11): 37.

[17] Wang Zhiqing. Discussion on factor analysis using "dynamic speed method" [J]. Finance and Accounting, 1988 (05): 55-56.

[18] Luo Shengli. Discussion on factor analysis with dynamic function method [J]. Finance and Accounting, 1987 (04): 43-44.

[19] Chen Yamin. Discussion on the basic principles of factor analysis [J]. Accounting Research, 1987 (01): 38-44+7.

[20] Cao Jinlin. A new method of quantitative calculation in factor analysis [J]. Finance and Accounting, 1986 (09): 27.

[21] She Minglong. Discuss the substitution sequence of chain substitution method [J]. Finance and Accounting, 1986 (05):
$42-45$.

[22] Bie Xingfa. Humble opinion on improving the factor analysis and calculation method of capital profit rate [J]. Accounting Research, 1986 (02): 47-49.

[23] Zhou Zonglin. Excerpts from the manuscript on the discussion of "Factor Decomposition Method" [J]. Accounting Research, 1985 (06): 43-47.

[24] Ji Liang. Discussion on the Factor Analysis Method in "Das Kapital" [J]. Accounting Research, 1984 (06): 39-40.

[25] Li Qiyu, Yu Rong. A new approach to factor analysis [J]. Accounting Research, 1984 (02): 25-29.

[26] Yang Zengwu. Questioning the scientificity of factor analysis in the index [J]. Statistical Research, 1984 (03): 41-49+56.

[27] Cho Se Jin, Bae Yun Jung, Kim Jong Min, Kim Donghyun, Baik Sung Hyun, Sunwoo Leonard, Choi Byung Se, Kim Jae Hyoung. Diagnostic performance of neuromelanin-sensitive magnetic resonance imaging for patients with Parkinson's disease and factor analysis for its heterogeneity: a systematic review and meta-analysis. [J]. European radiology, 2020.

[28] Science - Applied Sciences; Study Results from China University of Petroleum Broaden Understanding of Applied Sciences (Valve Internal Leakage Rate Quantification Based on Factor Analysis and Wavelet-BP Neural Network Using Acoustic Emission) [J]. Science Letter, 2020.

[29] Jinfeng Qiu. Study on the Analysis and Application of Aesthetic Value Model in Chorus Conducting Art Based on Factor Analysis [J]. Frontiers in Art Research, 2020, 2 (5).

[30] Feng Zhao, Qiang Yue, Junhao He, Yun Li, Heming Wang. Quantifying China's iron in-use stock and its driving factors analysis [J]. Journal of Environmental Management, 2020, 274.

[31] Meihua Chen, Liu Yun, Guifen Ma, Yonghong Yao. Factor Analysis Comparison of Factor Differences Between Old and New Calculation Methods [J]. Journal of Finance and Accounting, 2019, 7 (6).200-209.

[32] Liu Yun, Qiaowen Yang, Yonghong Yao, Guifen Ma. Current Situation and Existing Problems of Factor Analysis Method [J]. Journal of Finance and Accounting, 2019, 7 (6).192-199.

[33] Liu Yun, Yonghong Yao, Qiaowen Yang. New Solution of Factor Analysis Difference in Factor Product Combination [J]. Journal of Finance and Accounting, 2019, 7 (6).184-191.

[34] Fayçal Krim, Aicha Amer, Yousef Souar. Determine the Impact of the Related Party on the Financial Reporting Quality Using Factor Analysis - Case Study of Algerian Institutions [J]. International Journal of Finance and Accounting, 2018, 7 (5).

[35] Izolda Chiladze. Factor Analysis Aspects of the Enterprise's Operating Leverage [J]. Applied Finance and Accounting, 2017, 3 (1).

[36] Kang, Ho Young. Institutionalization of Management Accounting Change - Based on Institutionalization Factors Analysis of $\mathrm{ABC}$ in Chinese Companies - [J]. journal of Taxation and Accounting, 2013, 14 (6).

[37] ZHAO Shun-di, KONG Yu-sheng, LU Min. Mining cost analysis and cost control based on the sensitivity factor analysis [J]. Journal of Modern Accounting and Auditing, 2007, 3 (11). 
[38] Banghong Zhao, Zhenchuan Zhang, Lihua Guo. Factor analysis of the impact of changes in the appreciation rate of the asset appraisal [J]. Journal of Modern Accounting and Auditing, 2005, 1 (6).
[39] Mohamed Onsi. Factor Analysis of Behavioral Variables Affecting Budgetary Slack [J]. The Accounting Review, 1973, 48 (3). $535-548$. 